\title{
Subcortical White Matter Damage in A Patient With Early Stage Lupus Nephritis
}

\author{
Sin Yi Lyu ${ }^{1}$, Hsiang Cheng Chen ${ }^{2}$ and Chun Chi Lu*2 \\ ${ }^{1}$ Department of Radiology, Taiwan \\ ${ }^{2}$ Division of Rheumatology/Immunology and Allergy, Taiwan
}

Received: 眥: November 21, 2018; Published: 眥: December 04, 2018

*Corresponding author: Chun Chi Lu, Division of Rheumatology/Immunology and Allergy, Department of Internal Medicine, Taiwan

\begin{abstract}
Neuropsychiatric Systemic Lupus Erythematosus (NPSLE) refers to neurological or psychological manifestations, in which headaches and seizures are the most common clinical features. The most common lesions on brain Magnetic Resonance Imaging (MRI) of patients with NPSLE were white matter hyperintense lesions. Numbers and size of lesions were significantly higher in patients with lupus nephritis. This "case report" demonstrates the most common differential diagnoses of brain MRI imaging for patients with simultaneous NPSLE and lupus nephritis, including white matter disease, progressive encephalopathy and posterior reversible encephalopathy syndrome.
\end{abstract}

Abbreviation: NPSLE: Neuropsychiatric Systemic Lupus Erythematosus; MRI: Magnetic Resonance Imaging; LN: Lupus Nephritis; PRES: Posterior Reversible Encephalopathy Syndrome; DWI: Diffusion-Weighted Imaging; ADC: Apparent Diffusion Coefficient

\section{Introduction}

Neuropsychiatric Systemic Lupus Erythematosus (NPSLE) refers to neurological or psychological manifestations, in which headaches and seizures are the most common clinical features [1-2]. The most common radiographic findings in NPSLE include hyperintensity in the white or gray matter, parenchymal defects, and focal atrophy [3]. Hussein and his colleague highlight that the most common lesions on Magnetic Resonance Imaging (MRI) of patients with SLE were white matter hyperintense lesions and that number and size of lesions were significantly higher in patients with Lupus Nephritis (LN) [4]. We would like to emphasize the importance of integrating clinical manifestations, detailed neurological examination and MRI in the patient with early LN, which help identify brain lesions of NPSLE and prescribe adequate treatment.

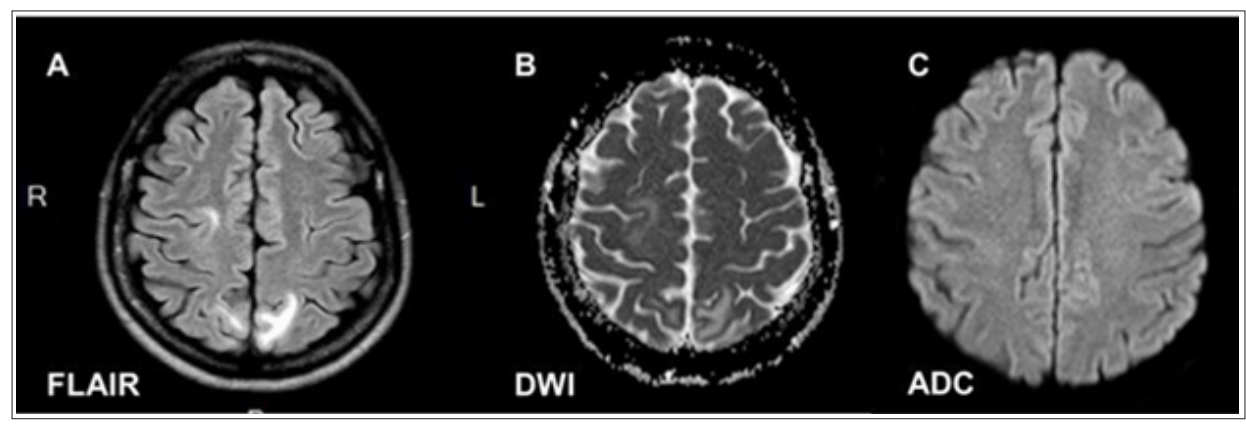

Figure 1: MRI of her brain demonstrated no vasculitis or venous thromboses, but multifocal hyperintense lesions in subcortical white matter in both frontoparietal lobes on Fluid-Attenuated Inversion Recovery Imaging (FLAIR) (A) Normal signal intensity on Diffusion-Weighted Imaging (DWI) (B) and hyperintensity on Apparent Diffusion Coefficient (ADC) mapping (C) were identified without contrast enhancement. 


\section{Case Reports}

We presented a 35-year-old woman was admitted after 1 month of fever and recurrent headache. Medical problems, drugs and chemical exposure were excluded. She had visited general practitioners before this admission, but intermittent headache continued. At our hospital, a blood analysis showed leukopenia, proteinuria, low serum complement levels and high serum levels of anti-double-stranded DNA, anti-ribosomal P, anti-Smith, anticardiolipin IgG, and lupus anticoagulant antibodies. Serum creatine level was $0.9 \mathrm{mg} / \mathrm{dL}$ with estimated glomerular filtration rate of $79.3 \mathrm{~mL} / \mathrm{min} / 1.73 \mathrm{~m}^{2}$. Urinal examination showed daily protein loss of $520 \mathrm{mg}$. Cultures did not identify any pathogens. Recurrent loss of consciousness and tonic limb posturing were observed after admission. Cerebral spinal fluid analysis showed normal glucose levels, increased intracranial pressure and increased protein levels in the absence of microbes. Electroencephalograms showed bilateral episodic slow theta waves without epileptiform discharges or spike waves. MRI of her brain demonstrated no vasculitis or venous thromboses, but multifocal hyperintense lesions in subcortical white matter in both frontoparietal lobes on fluid-attenuated inversion recovery imaging, normal signal intensity on Diffusion-Weighted Imaging (DWI) and hyperintensity on Apparent Diffusion Coefficient (ADC) mapping were identified without contrast enhancement (Figure 1) NPSLE was confirmed.

\section{Discussion}

NPSLE refers to neurological or psychological manifestations, in which headaches and seizures are the most common clinical features [1-2]. Patients with NPSLE should be determined whether these neuropsychiatric symptoms originate from separate diseases [5]. The most common radiographic findings in NPSLE include hyperintensity in the white or gray matter, parenchymal defects, and focal atrophy [3]. Differential diagnoses of brain MRI imaging for this patient include progressive encephalopathy and Posterior Reversible Encephalopathy Syndrome (PRES). DWI/ADC imaging did not show evidence of peripheral patchy diffusion. Neither hypertension nor visual loss developed. The patient received plasma exchange, pulsed methylprednisolone, azathioprine, and achieved good remission. Daily urinal protein loss was below 50 $\mathrm{mg} / \mathrm{dL}$ after treatment. Integrating clinical features, manifestations of brain MRI and neurological examination of our patient help define white marrow microstructure damage and distinguish with other common complications of LN, like hypertension related PRES. We should be more aware of white matter lesions in LN patients. Neurological examination and brain image check as routine followups are recommended for patients with medical history of LN even in early stages of chronic kidney diseases.

\section{References}

1. Unterman A, Nolte JE, Boaz M, Abady M, Shoenfeld Y, et al. (2011) Neuropsychiatric Syndromes in Systemic Lupus Erythematosus: A metaanalysis. Semin Arthritis Rheum 41: 1-11.

2. (1999) The American College of Rheumatology nomenclature and case definitions for neuropsychiatric lupus syndromes. Arthritis Rheum 42(4): 599-608.

3. Luyendijk J, Steens SC, Ouwendijk WJ, Steup-Beekman GM, Bollen EL, et al. (2011) Neuropsychiatric systemic lupus erythematosus: Lessons learned from magnetic resonance imaging. Arthritis Rheum 63(3): 722732 .

4. Hussein MA, Elsabagh YA, Hosny A, Elgendy H (2017) Silent cerebral MRI findings in lupus nephritis patients: Is it clinically significant? J Adv Res 9: 63-67.

5. Gulinello M, Wen J, Putterman C (2012) Neuropsychiatric Symptoms in Lupus. Psychiatric Annals 42(9): 322-328.

\section{ISSN: 2574-1241}

DOI: $10.26717 /$ BJSTR.2018.11.002142

Chun Chi Lu. Biomed J Sci \& Tech Res

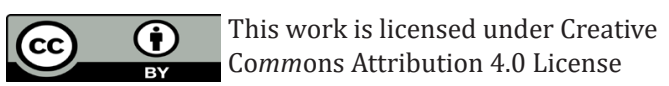

Submission Link: https://biomedres.us/submit-manuscript.php

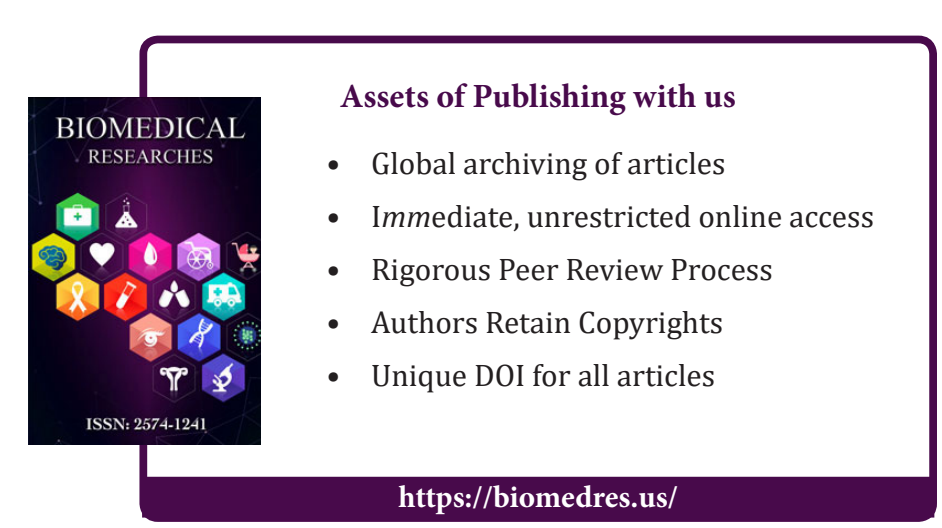

